\title{
Revealing Phenomena of Heat Energy, Levity, Gravity and Photons Characteristic Current to Light on Dealing Matter to Sub-Atom
}

\author{
Mubarak Ali \\ Department of Physics, COMSATS Institute of Information Technology, \\ 45550 Islamabad, PAKISTAN \\ mubarak74@comsats.edu.pk or mubarak74@mail.com, Ph. +92-51-90495406
}

\begin{abstract}
Technology has almost reached to its climax but the basic understanding of science in many phenomena is still awaited. Scientific research reveals strong analogy between electron and photon. Atoms that execute electronic transitions, on absorbing heat energy, excite electrons. Deexcitation of electron results into depicting energy in the shape of Gaussian distribution and where inertia is involved. The wavelength of photon at point of generation remains in inter-shell distance and atoms of all those elements that glitter perform like magician, throwing one and catching other, where an electron excites at shunt energy and configure trajectory under levity and de-excites at free fall configuring trajectory under gravity and silicon atom is a model system. In band gap of such atoms, heat energy of merged photons is cultivated and that shunt energy perturb the balance of inherent energy between electron and nucleus, which is not the case in atoms do not glitter. Uninterrupted confined inter-shell motion of electron results into photon that can travel immeasurable length. Such photons increase wavelength on decreasing frequency, propagate to hard X-ray, to visible spectrum, and to beyond. Here, I discuss that heat energy is due to merged photons, current due to photons wavelength in inter-shell distance and light photons wavelength in visible spectrum. Force of repulsion or attraction in certain materials engages phenomenon of levitism or gravitism instead of magnetism where inertia is exempted. All structural motifs and dynamics are subject to characteristic photons. A structural design delivers straight-forward application on coordinating overt photons or merged photons. The various gadgets, devices and instruments only operate energy as per need of necessity. Here, materials science explores matter to sub-atom while coordinating energy and devises science to describe.
\end{abstract}

Keywords: materials science; energy; materials chemistry and physics; nanoscale phenomena; atomic scale phenomena; sub-atomic scale phenomena

\section{INTRODUCTION:}

Humanity is being benefited by heat energy and light since the existence and electrical phenomena have been studied since antiquity. Catching fire in various stuffs is a usual phenomenon known since antiquity. Everyone is taking benefit of those blessings but the clear understanding behind those phenomena is hazy and their coordination to various sorts of matters is peculiar.

A large number of studies are available in the literature, dealing with light-matter interaction, and it has been covered largely under a phenomenon, namely, surface plasmons. Origin of plasmons was explored in some early published reports [1-4]. A plasmon is a quantum of plasma oscillation and represents the collective oscillations of the free electron gas density.

The interaction of light (photon) to matter is recognized in the form of various terminologies, namely, phonon, excitons, and plasmon, etc. Recently published review discusses the lightmatter interaction by taking into account properties of polaritons modes in twodimensional materials applications having certain range of the spectrum [5]. The concept of excitons (electron-hole pairs) was first proposed by Frenkel [6] and deals with excited state of an atom in a lattice travelling in particle-like fashion without net transfer of charge. Excitons can be formed on absorption of photon by a semiconductor (quantum dot) [7]. A phonon is a collective excitation in a periodic, elastic arrangement of atoms or 
molecules in condensed matter and their concept was first proposed by Igor Tamm in 1932 [8].

$A$ vast number of studies deal with formation process of tiny-sized particles -structure evolution at nanoscale in those atoms. The tiny-sized cluster is a simple chemical compound which has a variety of important applications in diversified areas [9]. The unique nature of nanocrystals solicits fabrication of new materials having controlled features [10]. The likely development of nanoparticles technology is an obvious long-term benefit [11]. With the success of assembling colloidal matter in a useful structure, the atoms and molecules will also be treated as material [12]. The investigation of the dynamics of an individual nanoparticle should be taken as a prime concern prior to going for further solid deliberations [13]. A good understanding on the surface features of nanoparticles will lead into development of higher order materials [14]. Tiny-sized clusters possess molecular-like electronic structures and non-fcc geometric structures [15] and chemical properties of gold nanoparticles change with size [16]. A variety of strategies to process material in different length scales have been introduced and discussed in the literature by keeping in view particular emphasis on the shape and size. From another perspective, research in the field of optics, radiations, photonics and light is also on the way and growing rapidly.

It has been suggested that localized dynamics of the process is the main cause that helps to evolve the structure of tiny-sized particles of gold [17-21], silver [21], and carbon $[22,23]$. Under the process of synergy, electron states of atoms of tiny particles are diffused as per available room [17]. A tiny-sized particle where it doesn't have a specific geometry, it goes for packing and coupling under nonuniform drive and not all bound atoms possessed directional interactions. Thus, their atoms reveal various sorts of electrondynamics including diffusion of electron states depending on the process of synergy. Overall, atoms of such tiny particles partially deform or stretch depending on the process of synergy. However, where impinging of electron streams is regular, the underlying atoms of monolayer tiny-sized particle reveal diffusion of electron states, which is orientation based [17]. When the diffusion of electron states of an atom is orientation based, we say stretching or onedimensional elongation, and when it is nonorientation based, we say deformation as discussed in several studies [17-23]. In both cases, where either impinging of regular electron streams are not for fixed period with respect to underlying atoms of monolayer tiny particle or the structure of monolayer tiny particle is not in two-dimension, the diffusion of electron states can be non-orientation based. Their electronic structures do not modify into smooth elements on propagating photons of adequate energy and same is the case, on packing, into large-sized particles as discussed elsewhere in the case of gold lattice [18-21]. The same is the case in silver lattice [21]. Additionally, the same mechanisms of atomic deformation and stretching was observed in synthesized tiny-sized grains of ultrananocrystalline carbon films or nanocrystalline carbon films [22], and also discussed elsewhere [23]. Again, monolayer tiny particle geometry in rhombus shape was chosen to explain how the atoms of electronic transitions bind, and when uniform photon couplings take place, stretching and deformation of its atoms, and where diffusion of electron states is orientation-based, their modifying into smooth elements under adequate energy of propagating photons as given elsewhere [24].

In this work, I have discussed those overt photons that coordinate medium while interactions, change them, into merged photons or squeezed ones, they are heat energy, and their absorption by atoms capable of executing electronic transitions resulting into photons characteristic current. Then atoms that do not execute electronic transitions split under the application of field of photons characteristic current (photonic current) resulting into formation of impulsive terminals, hence, switching of photons characteristic current to ones increasing wavelength or decreasing frequency. Here, I discuss the charisma of an atom, which is capable to execute electronic 
transitions under controlled electron-dynamics and while restricted to phenomenon of elastically-driven electronic states. The analogy between electron and photon is explained by taking silicon atom as a model system. Structural response and relevance to various forms of energy is discussed as well.

\section{RESULTS AND DISCUSSION:}

Under adequate amount of energy, which is due to photons characteristic current, inert gas atoms split into electron streams and nucleus. Those electron streams transferred gained energy to underlying atoms of tiny particles, on impingement. Atoms during electronic transitions remain positioned while in tiny-sized particle. The impinged electrons are ejected from the inert gas atoms under the field of photonic current [25]. Thus, impinged electrons to underlying atoms of tiny-sized particle possessed discrete energy feed by photons characteristic current. Regardless of that inert gas atoms possess filled electron shells, they intake photons characteristic current as they transferred into their band gaps via inter-shell distance, that's why, forced out electrons are ejected in the form of streams. This is not only in atoms of inert gases but in hydrogen atoms and other suitable materials capable of forming so-called plasma, as well. On splitting of atoms, electrons are transferred to underlying atoms capable of executing electronic transitions, for example, gold, silver, carbon, etc., as a result, electron states of atoms are diffused in the orientations of transferred energy. Such atoms deform or stretch depending on the mode of impinging electron streams (also the process of synergy) along with their positional orders in tiny-sized particle. Where atoms of monolayer one-/two-dimensional structure elongate in the same direction, electron states of those atoms are also diffused in the same orientation. Simultaneously, at electronphoton-solution interface, photons acquired wavelengths in the range of hard $\mathrm{X}$-rays and utilized energy to modify suitably stretched atoms of those structures into smooth elements [17]. The phenomena of deformation and stretching of single atom positioned in tiny-sized lattice along with coordination of hard X-rays photons are shown in Figure $1\left(a_{1}\right)-\left(a_{6}\right)$ and in the case of one-dimensional array of three atoms shown in Figure $1\left(b_{1}\right)-\left(b_{3}\right)$. Photons wavelength in hard X-rays possess energy to create interspacing distance of smooth elements in the range of $\sim 0.1 \mathrm{~nm}$ to $\sim 0.2 \mathrm{~nm}$ as has been discussed elsewhere [17-22].

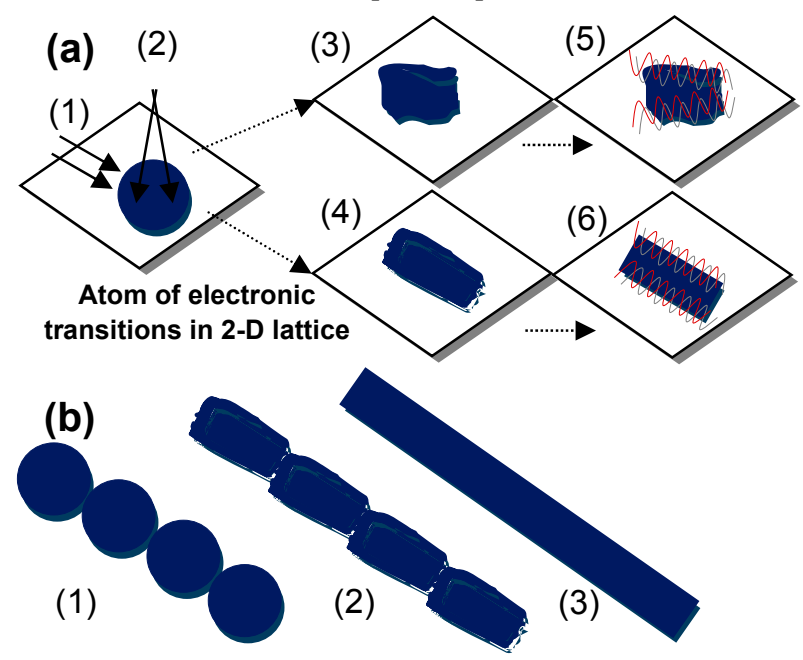

Figure 1: Impinging electron streams to atom positioned in monolayer two-dimensional lattice at (a $\left.a_{1}\right)$ fixed (and same) angle, ( $\left.a_{2}\right)$ different angles, (a) atom deformed, $\left(a_{4}\right)$ atom stretched, photons of hard X-rays (a5) not modified electronic structure of atom deformed, (a6) but modified electronic structure of atom stretched (into smooth element) and $\left(b_{1}\right)$ one-dimensional array of atoms, $\left(b_{2}\right)$ onedimensional array of stretched atoms and $\left(b_{3}\right)$ their smooth element on propagate hard $X$-rays photons.

In merged photons (or squeezed ones) rather than overt photons propagate they have been shaped in different forms as per their interaction and coordination of medium. Instead of photons with definite wavelengths (overt photons), they are more in tits and bits. Due to friction, their energies have been transformed into heat energy and they are termed 'merged photons' or 'squeezed photons' or they are simultaneously merged as well as squeezed ones. Such heat energy can't be absorbed by the inert gas atoms, however, atoms of electronic transitions are capable to absorb (intake) such heat energy in their band gaps and do initiate excitation (s) of electron (s). Thus, energy of merged photons (or squeezed ones) is related to heat energy and is transferred to medium (atoms) on coordination, 
through propagation. Such heat energies, on propagating into band gap of atoms, do not diffuse the electron states but instead excite electrons under the shunt energy required by an electron at target. Diffusion process of grains and atoms in various materials under steady state has been explained by the Fick's laws. But the diffusion of electron states of atoms execute electronic transitions remained unexplained as electrons transferred the energy on split of certain nature atoms (inert gas atoms) [17]. Marconnet et al. [26] reviewed in details the heat conduction phenomena in carbon nanotubes and related nanostructured films. Merged photons (or squeezed ones) of different marks of associated energy coordinate a variety of medium are shown in Figure 2 .

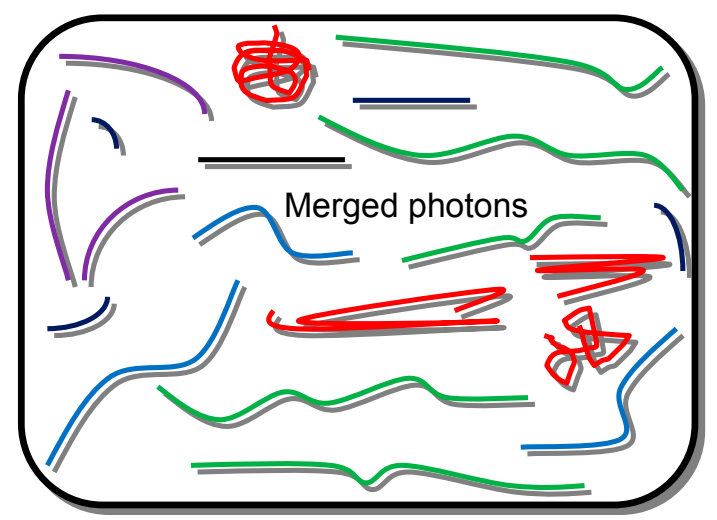

Figure 2: Different Merged photons (or squeezed ones) coordinate various mediums reveal different signatures of their associated heat energy.

As discussed, where impinged electron streams to atoms of one-dimensional arrays are at fixed angles, diffusion of electron states of atoms is in the orientation of impingement, as a result, atoms elongated one-dimensionally and modified into smooth elements under the application of hard X-rays photons. In the case where atoms do not form one-dimensional arrays of structure, impinged electron streams deform them, and electron states of atoms are diffused non-orientationally. These phenomena are clear, observable and understandable when atoms of electronic transitions are configured in tiny-sized particles. Atoms that standalone reveal different behaviors under the process of synergy. Obviously, the drive of single atom under attained dynamics in the medium is zigzag where diffusion of electron states will not take place orientationally, thus, that deformed atom can't modify into smooth elements on propagating photons at surface.

When heat energy of merged photons (or squeezed ones) propagates in the band gap of an atom, let us say silicon atom, and reaching upto shunt level, an electron is excited to higher state. As observed in solar panel at $45^{\circ}$ angle (approx.) with respect to plane oriented to the south (Figure 3) will result into an average maximum power generated throughout the year where configured lattice of silicon atoms not only absorbed the heat energy of merged photons but also dealt direct absorption of radiations, thus, provided bonus shunt energy to band gaps of those atoms. However, the latitude of the geographical location is the key where solar panels should be mounted along with time of the year. That bonus energy excited electrons at faster rate and shunt energy to excite electrons of atoms is involved for less time. A merged photon (or squeezed one) may not deal discrete amount of energy required to excite an electron and it can only be excited on reaching energy at threshold level called shunt energy. However, in silicon atom, the probability of electronic excitation becomes higher resulting into greater length of generating photon, thus, effective utilization as a current.

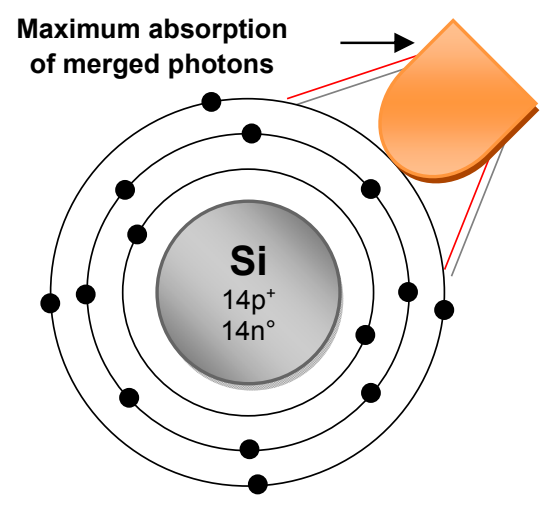

Figure 3: Silicon atom shows favorable orientation with respect to reside plane to absorb maximum compatible energy of merged or direct photons resulting from sunlight throughout the year.

Electron-dynamics are restricted to only valence shells of an atom executing electronic 
transitions. To generate photons characteristic current, the fluctuations of electrons remained coherent within two shells. The cycles of excitation and de-excitation of electrons in silicon atoms are at fast rate and non-stop for longer period. On titling silicon solar panel at certain orientation with respect to base results into varying the efficiency, which is quite effective in the peak hours of sunlight.

In Figure 4 (a), transition of single electron in the inner most shell of silicon atom is shown where excited to one state higher as indicated by upward arrow and energy configured in the trajectory of electron, when electron was deexcited under its free fall, its trajectory was again configured with equal amount of energy but inverse to one when excited. This is a complete cycle of electron, which results into frame energy in Normal distribution shape. Deexcited electron is denoted by the grey color and excited position is in blank dot. Both excited and de-excited states of an electron are indicated by arrows along with shunt energy required to excite it. The shunt energy required to re-excite the same electron is also labeled. Another electron is also shown in Figure executing the same phenomenon as discussed above but in the middle shell where the shunt energy is indicated in rearward. Continuous phenomenon of excitation and de-excitation of electron within inter-shell results in an unterminated photon wavelength equal to intershell distance, at the point of generation. As further clarified in Figure 4 (b) where only single electron was considered (in black color), which excited under absorbed energy at shunt level as indicated by arrow. The potential energy of excited electron is the maximum on reaching to higher state and on restoring back to original position (lower shell) under gravity the kinetic energy becomes the maximum. However, the energy configured at the trajectory of excited electron is due to levity (anti-gravity/negative gravity) as frictional force is diminished at such level (levity of electron). The process of self-returning of same electron to original state after completing 9 cycles shaped the energy like wave as in Figure 4 (b). The same process takes place in many atoms of the lattice and many electrons of the atoms result into generating photons characteristic current. The filtering of heat energy of merged photons (or squeezed ones) at shunt level, which is required to excite same electron 9 times is shown in Figure 4 (b). De-excited electron will arrive at same point from where it is excited and due to topology of an atom, thus, generating photon on completing each cycle is pushed back having no coordination to the ones generating by other electrons of the atom.

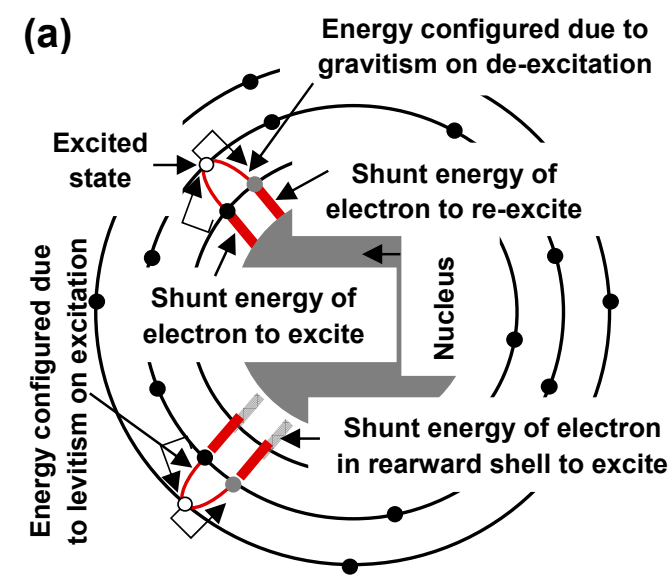

(b) Unterminated photon, wavelength in

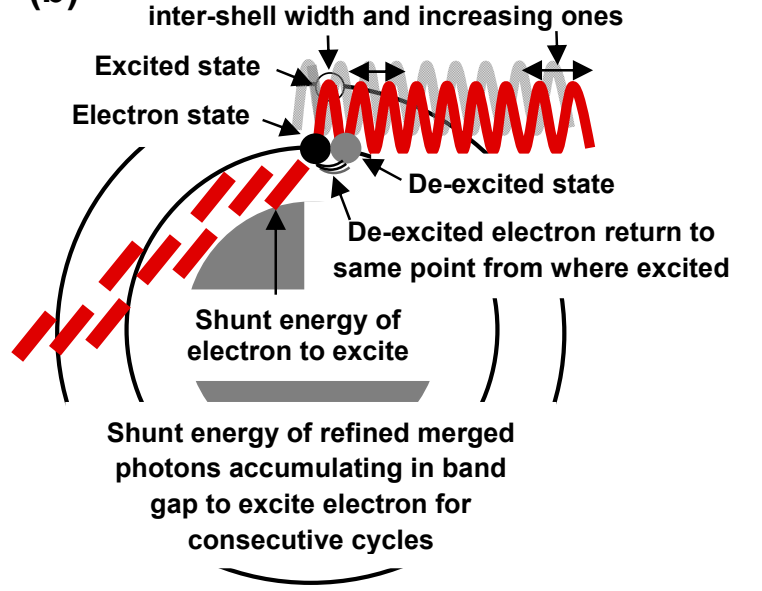

Figure 4: (a) Excitation and de-excitation of electron in silicon atom and (b) repeated process of excitation and de-excitation of same electron where photon is increasing wavelength with respect to propagation distance and absorption of shunt energy to re-excite on completion of each cycle.

An atom builds electronic shells or orbits in three-dimensional space where configuring of energy under its controlled electron-dynamics is in trajectory like Gaussian distribution shape connecting every time while re-excitation of de- 
excited electron. On de-excitation, an electron comes into same level of natural force of nucleus possessed prior to excite. However, continued supply of shunt energy to that electron in each cycle of de-excitation to reexcite will lengthen the wave. Bohr proposed that electrons move around the nucleus in allocated orbits where they have fixed energy as long as atom is in ground state. Thus, to excite an electron, it requires a fixed amount of energy and word 'orbit' is also suitable to quote.

An electron is required to excite from filled shell of silicon atom, one by one, to transfer shunt energy in band gap and when one electron from the outer filled shell is excited, the excitation in the inner most shell is regulated. The regulation of excitation in suitable atoms depends on the number of filled shells as shunt energy deals with all those entering the band gap. When an electron goes to excited state, the gap of entering energy (shunt level) is automatically formed as filled shell also become unfilled. Those entered energy, each in shunt level of electron, are utilized only to excite electrons, on targets, and have nothing to do with made trajectories against excited/de-excited electron. This makes an atom to perform like magician due to disturbed electron. The made trajectory is due to energy of levitism as shown in Figure 4 (a), when electron excited from the middle shell. The configuring trajectory while de-exciting electron is because of gravity due to achieving stability. While exciting, an electron is arriving toward relaxation, while de-exciting it is moving toward ground state and atom is in non-isolated system. In this scenario, trajectory configured energy at left-side (while exciting an electron) is due to levity (negative gravity or anti-gravity) and trajectory configured energy at right-side (while de-exciting an electron) is due to gravity (positive gravity) and both together translate energy having nature of wave and when in inter-shell distance, it is said photon characteristic current. Thus, in various socalled magnetic materials, the force of repulsion and attraction is not due to magnetism but relates to levitism phenomenon and gravitism phenomenon, respectively, where inertia is exempted due to intrinsic nature of those materials, for example, in iron. The force of repulsion is due to levitism phenomenon while force of attraction is due to gravitism phenomenon. The levitism phenomenon is due to energy (force) of levity or negative gravity or anti-gravity, whereas, gravitism phenomenon is due to energy (force) of gravity or positive gravity or anti-levity. Thus, so-called magnetic behavior of materials incites the Coulomb's Law.

Sir Isaac Newton explained the gravity in Newtonian Physics. There is no concept of anti-gravity in Theory of General Relativity as explained by Sir Albert Einstein. When an electron excited, it changed the state where potential energy (P.E.) was the minimum and kinetic energy (K.E.) was the maximum, on reaching to higher state changed the state again where P.E. was the maximum and K.E. was the minimum. When that electron deexcited, it changed the state where P.E. was the minimum and K.E. was the maximum, on reaching to ground state, changed the state again where P.E. was the maximum and K.E. was the minimum. But that electron excited again under shunt energy given to it and changed the state where P.E. was the minimum and K.E. was the maximum, thus, will repeat the process of excitation and deexcitation again. All those changed states of the electron involved inertia and resisted its motion, were the cause of configured energy in propagating wave and by restricting electron motion within inter-spacing of shells (orbits).

In Figure 5 (a), atomic configuration of argon atom is shown where absorbed energy is due to photons characteristic current and not due to merged photons (squeezed ones) as they are rarely propagated into band gap of inert atom. The capability to absorb heat energy from surrounding is related to emptiness of valence band of an atom, which is not in the case of inert gas atoms because they own filled outer most shell as well. Helium atoms may absorb heat energy where merged photons (or squeezed ones) own wavelengths close to photons characteristic current. Now, $\mathrm{H}$ atom breaks as well while applying suitable field of photonic current and this is why it is always in 
molecular form where probability to propagate photons characteristic current is small. Such atoms split into electron streams and nucleus under the appropriate field of photonic current as photons characteristic current possess wavelength equal to inter-shell width. Nevertheless, split of atoms switch the field of photonic current into increasing wavelength of photons by in-situ employing their intrinsically made gap i.e. electron streams and nucleus as discussed elsewhere [25]. The phenomenon can be revealed in Figure 5 (b) where photons characteristic current break argon atoms, on propagating into band gap, thus, create gap, through which switch into photons increasing wavelength - to X-rays, to ultraviolet, to visible spectrum and till collapsing the energy into merged photons (or squeezed ones).

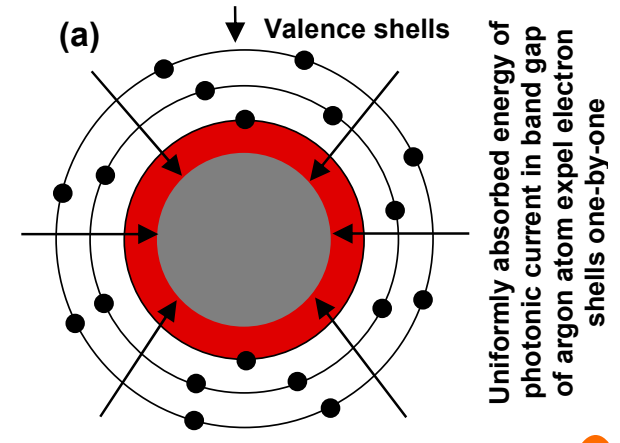

(b)

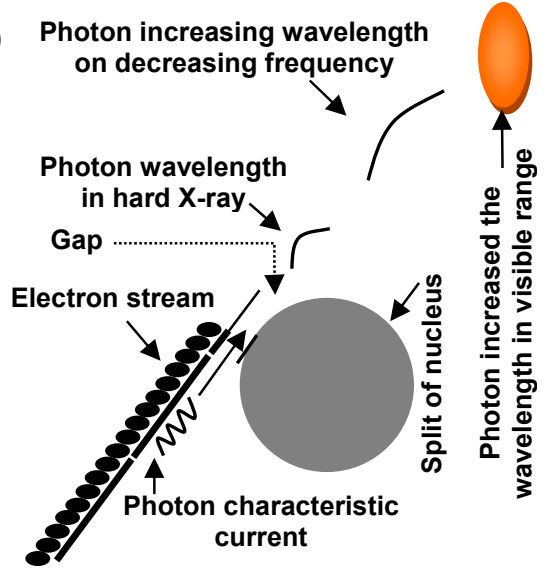

Figure 5: (a) Band gap of argon atom absorbed energy of photonic current and (b) split into electron streams and nucleus where created gap switch wavelength of photons characteristic current into the increased ones.

When a certain designed material is introduced to deal photonic current, for example, connecting tungsten filament of bulb between so-called negative terminal (wire where photonic current is propagated) and positive terminal (where surplus photonic current is available) result into light, mainly, as the coil setup is under maintained vacuum, and in the case of open air setup, so-called electric heater where photonic current is mainly converted into heat. The same is the case in all household appliances (LCD, tube light, TV, Radio, microwave oven, etc.) and according to in-built features of the certain appliance, different utilization of photonic current are possible. In the case of silicon solar panels and similar kinds of gadgets, utilization of the inverters only modify the lengths of photons characteristic current so that enabling their propagation in randomly distributed wires of the home. When a device breakup, the propagation of photonic current is also halted where positive terminal is no longer remained effective and it is involved to utilize excess photonic current into next appliance connected in series or to propagate back.

An electron has mass and it is diffused (penetrated) on deformation or stretching of occupied atom. In the case of photon, it doesn't have mass and it involves only discrete energy. In this context, overt photons neither diffused nor impinged and they even don't travel to strike, they either propagate or their energy is being absorbed by the coordinated (or interacted) matter/medium. Thus, under certain amendment overt photons alter into merged photons or squeezed photons. Consequently, they are phonons under certain coordination or interaction where frictions involve. Thus, merged photons (or squeezed ones) are heat energy -phonons. A photon having certain wavelength when coordinates to certain medium evolve into shape depending on the characteristic of matter in that medium. The nature of coordinated photon itself signified as its energy reveals different behavior; bounce back, propagate, transmit, absorb, evolve into the shape of that matter, reabsorption, etc. Photons having controlled wavelength in visible range enable resolving the image down to 0.2 $\mathrm{mm}$-a magnification that human eye can see. Photons having controlled energy in the wavelength of $X$-rays propagate through human body and identify the signature of 
fracture. Photons characteristic current propagate in suitable wire, thus, they are photonic current. Different cameras and devices manipulate the image on screening (copying) reflected back photons to an object.

When it is said that an electron is spoiled it doesn't mean that that atom is ionized but it means that there is no more elastically-driven behavior left for that electron and that atom enters in the zone where electron is executing plastically-driven behavior, thus, that atom either deformed or stretched. When atom is an atom, it is a powerful device functioning like sun but at atomic level, when an atom stretched, depending on rate of diffusing electron states and number of electrons, different application may emerge, for example, propagation of photons wavelengths in hard $X$ rays where smooth element of electronic structure is made. In the case of diffusing electron states non-orientationally, electronic structures of atom are viable for various catalytic roles, last but not least, when an atom erodes, it may still be useful for certain application where needs to be explored. This explanation is eligible for atoms of all elements that execute electronic transitions.

In various ultrananocrystalline carbon (UNCC) and nanocrystalline carbon (NCC) films their field emission application as well as display panel applications are due to forming of smooth elements of tiny grains. In very small size of the cluster, the most of the atoms shape their structure in two-dimensional lattice, they stretch on diffusing electron states orientationally and propagating photons wavelength in hard X-rays modify electronic structure into smooth elements as discussed such phenomena elsewhere [22, 23]. Those tiny grains of UNCC/NCC films modified electronic structures into smooth elements are the source of photon field emission and display panel applications (not electron field emission). As photonic current propagates inside interspacing distance of smooth elements made of tiny grains, the wavelength of photons characteristic current reach in the range of $X$ rays on termination of those channels, thus, they are utilized straight-forward for photon field emission or display panels application.
The performance of such UNCC/NCC films should be notified in terms of formation of maximum smooth elements of tiny-sized grains followed by the nature of their structural connects and such films give phenomenal controlled features of the emitted fields. The same approach should be considered in studying phenomena like surface enhanced Raman scattering or localized surface Raman spectroscopy of nanoparticles [27].

Distribution of heat energy in disordered structure is non-uniform. More heat is resulted in structures where atoms are randomly distributed as photons characteristic current dissipate within such structure, they mingled to each other, crossing, overlapping, thus, raise the temperature (heat energy) of lattice. Thus, they again transform into merged/squeezed photons, act as feed to excite an electron, thus, repeated cycles of generating photons via electron-dynamics and conversion into merged photons further distort the lattice initially in disordered state. Similarly, when photons of different wavelengths coordinated disrupt medium, they spread heat energy in the form of merged photons. When interactions of photons to matter are at greater energy, heat is dissipated in greater extent as well. Matter assigns different roles to photons on interaction, converting one form of energy to another, depend on the structure and topology. In Bragg's diffraction, amorphous materials don't reveal any specific structure on interacting to photons wavelength in X-rays. Photons vary wavelength is a cycle of energy where in some cases they work in the form of heat energy (merged photons) and enable the excitations of electrons in atoms of electronic transitions, on de-excitations of those electrons and repetition of the phenomenon result into generate photons characteristic current, which propagate in suitable wires, thus, working as a photonic current. In some cases, photons having characteristic of current are utilized to break the matter where atoms do not possess phenomenon of electronic transitions as in the case of inert gas atoms and eject electron streams, thus, they are utilized to deform or stretch atoms of electronic transitions. 
At some instant due to not propagating photons as well in a region of screen in my computer (ProOne400) as in the remaining regions of desktop screen, which may be due to several times shut off/shut on the current of my PC, some words of the text in this manucript are not revealing exact words as shown in Figure 6 (a) and while capturing the image through Print Screen option. However, on justing moving the cursor on same region of text the words of the text became readable as shown in Figure 6 (b). This again confirm that current we use in our daily life -in all walks is due to propagation of photons wavelength in inter-shell distance instead of flow of electrons as discussed elsewhere [25].

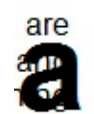

st.

2d it

ut it

iven

Itom

Iting

itom

s an should be notified in terms of formation of xımuim smooth "elements" of 'tiny-sıżed" grains followed by the nature of their structural oririecist- ariut sucti filrs give phenomenal controlled features of the emitted fields. The

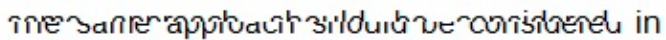
studying phenomena like surface enhanced

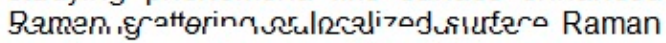
spectroscopy of nanoparticles [27].

ribution of heat energy in disordered structure is non-uniform. More heat is resulted in

should be notified in terms of formation of maximum smooth elements of tiny-sized grains followed by the nature of their structural connects and such films give phenomenal ed it controlled features of the emitted fields. The ut it same approach should be considered in iven studying phenomena like surface enhanced itom Raman scattering or localized surface Raman Iting spectroscopy of nanoparticles [27].

itom Distribution of heat energy in disordered 5 an structure is non-uniform. More heat is resulted

Figure 6: Screen print of (a) damaged words text and (b) undamaged words text by just moving the cursor on text.

The set modalities of all sorts of photons depend on the origin of their generation establishing roles set by the manufacturer or expert while coordinating characteristics matters. In this context, structural design is crucial in targeting the specific application of certain material and many studies are now targeting and exploring structure either standalone or in relation to other fields of sciences [28-38]. As discussed, uniform photon couplings take place in materials evolved in one-dimensional or two-dimensional structure $[24,32]$ and non-uniform photon couplings in materials evolved in three-dimensional structures [32]. To measure temperature of such structures in various materials having selective size and medium is integral part to understand science of different behaviors and some of the studies throw light on that [39-41].

On moving optical tweezers in real-time control system, tunable arbitrary geometries of cold neutral atoms for quantum engineering are prepared [42] and regular arrays of individual controlled cold atoms as well [43]. A recent study explained the role of van der Waals interactions in the case of isolated atom [44] and they arise from induced dipoles, which can be attained when fluctuations of charge density are in wavelike nature [45]. As discussed in several investigations that structural motifs are owing to dynamics that develops under localized conditions of the processes [17-25] and under the virtue of heat energy, photons of certain wavelength and then photons characteristic current, thus, to explain them prior to or along with dynamics and structural motifs is in scientific spirit. They are at forefront in design of any sort of material having certain application and without the involvement of photons (heat energy, current and light) it is not possible to design even ordinary featured structure along with interplay of its properties. All that glitters need not be Au but TiN or ZrN as well [46]. However, all elements and compounds glitter where atoms execute electronic transitions, and what is needed is to attain shunt energy to excite electron under controlled dynamics. It is possible to measure temperature of atoms, their nanoscale components and at bulk scale as well without the involvement of thermocouples and introducing various gadgets, theories, etc. What is required is to determine shunt energy to excite an electron of atoms with respect to normal living temperature. As long as atoms of electronic transitions are at ground state their electrons remain confined in shells or orbits and availability in the form of gas density is not understandable. In the case of Drude model that large number of electrons in metals is as electron gas, freely moving in metal, and 
become the cause of positive charge. In case we consider their source outside then they are contaminants and should not be the case of an atom as it is the purest form of the matter, then how an inactive matter diffused inside those atoms, is again not understandable. The oscillation of that electron gas is called plasma oscillation and quanta of this charge density oscillation are called plasmons. In fact, heat energy of suitable merged photons transferred to band gaps of atoms and is referred to localized heating of atoms at which electrons excite.

\section{CONCLUSIONS:}

In various materials, structural adjustments to different forms of energy find the way out for certain application, indicating that design of the material manipulates energy for targeted application. Sun is source of energy to earth, operates matter as per built-in characteristic, humanity is just exploring those traits and benefiting for their efforts.

Photons characteristic current, when it collectively propagate in suitable wire, is called photonic current, such photons generate under natural confinement of electrons. On coordination of photons having various wavelengths to mediums their energy reshape into merged photons or squeezed ones called heat energy -due to involvement of friction where energy of wavelength is collapsed. Such heat energy is filtered by atoms of electronic transitions depending on their electronic configurations. In band gap of an atom, the shunt energy excites an electron to higher state. When atoms of electronic transitions absorb energy in band gap, the natural binding energy of electron and nucleus is perturbed in discrete manner; it enables electron one position higher where it configures trajectory due to energy of levity and when it de-excites under free fall trajectory configures due to energy of gravity. The photon characteristic current is generated as long as atom controls electron-dynamics under given shunt energy in each cycle of electron and connecting of Gaussian distribution shapes configure the shape of energy like wave propagating rearward normal to inter-shell motion of electron. At each state of changed motion of electron, inertia is involved. An electron owns strong analogy to photon in a manner that intershell motion of an electron result into configures energy of surrounding in discrete manner having shape like a wave.

Structure of certain materials deliver straightforward application on interacting and coordinating merged photons, photonic current or light, for example, heat energy keep warm to many living insects where light and current is not accessible. In solutions of various metallic colloids and quantum dots, on interacting light, split into colors and in ultrananocrystalline and nanocrystalline carbon films photonic current switch into field emission, flat-panel display, etc. In certain materials, the forces of repulsion or attraction is due to levitism or gravitism instead of magnetism and incite Coulomb's Law, when inertia involved due to intrinsic nature of certain atoms, the resulted behavior of an electron deal levitism-inertia-gravitism phenomenon and responsible of propagating energy -called photon, which incites electromagnetic spectrum at increasing wavelength. All suitable atoms of electronic transitions are naturally built-in machines (devices) to filter heat energy of merged photons into the most valuable asset of modern life existing on earth and atoms of silicon lattice are the best choice till to-date. The matter is nothing without energy and all matters operate under its functions reaching from the sun in the form of radiations and light, mainly, and living organisms in the smallest sizes require that energy to survive. An electron is also matter, perhaps, the smallest entity of matter available on the earth and it is most valuable when it works within natural confinement (domain).

Clearly, electrons are matter, occupy space, possess mass and impinge (strike) under given energy from external source while they excite/de-excite from internal source. Whereas, all types of photons possess energy but not mass, they occupy space to propagate and spread but not sort of impinging (striking) or exciting/de-exciting entities. Atoms are bound together under the energy of their own photons provided from the internal source (shunt energy accumulated in band gap) but stretch in the 
lattice under the action of diffusing electron states, hence, indicating their stretching (compression and aligning) under the modified energy of their electrons supplied from the external source (under impingement of regular electron streams, which detached on split of inert atoms, or under the process of synergy).

\section{References:}

[1] D. Bohm, D. Pines, A Collective Description of Electron Interactions. I. Magnetic Interactions, Phys. Rev. 1951, 82, 625-634.

[2] D. Pines, D. Bohm, A Collective Description of Electron Interactions: II. Collective vs Individual Particle Aspects of the Interactions, Phys. Rev. 1952, 85, 338-353.

[3] R. H. Ritchie, Plasma Losses by Fast Electrons in Thin Films, Phys. Rev. 1957, 106, 874-881.

[4] D. Bohm, D. Pines, A Collective Description of Electron Interactions: III. Coulomb Interactions in a Degenerate Electron Gas, Phys. Rev. 1957, 92, 609-625.

[5] T. Low, et al., Polaritons in layered two-dimensional materials, Nat. Mater. 2016, doi:10.1038/nmat4792.

[6] J. Frenkel, On the Transformation of Light into Heat in Solid. I, Phys. Rev. 1931, 37, 17-44.

[7] O. D. D. Couto Jr. et al., Charge control in $\mathrm{InP} /(\mathrm{Ga}, \mathrm{In}) \mathrm{P}$ single quantum dots embedded in Schottky diodes, Phys. Rev. B 2011, 84, 125301-7.

[8] https://en.wikipedia.org/wiki/Phonon.

[9] M. Brust, M. Walker, D. Bethell, D. J. Schiffrin, R. Whyman, Synthesis of Thiol-derivatised Gold Nanoparticles in a Two-phase Liquid-Liquid System, J. Chem. Soc., Chem. Commun. 1994, 801-802.

[10]R. L. Whetten, J. T. Khoury, M. M. Alvarez, S. Murthy, I. Vezmar, Z. L. Wang, P. W. Stephens, C. L. Cleveland, W. D. Luedtke, U. Landman, Nanocrystal Gold Molecules, Adv. Mater. 1996, 8, 428-433.

[11] M. Brust, C. J. Kiely, Some recent advances in nanostructure preparation from gold and silver particles: a short topical review, Colloids and Surfaces A: Physicochem. Eng. Aspects 2002, 202, 175-186.

[12]S. C. Glotzer, M. J. Solomon, Anisotropy of building blocks and their assembly into complex structures, Nature Mater. 2007, 6, 557-562.

[13]S. Link, M. A. El-Sayed, Shape and size dependence of radiative, nonradiative and photothermal properties of gold nanocrystals, Int. Rev. Phys. Chem. 2000, 19, 409- 453.
[14]C. P. Shaw, D. G. Fernig, R. Lévy, Gold nanoparticles as advanced building blocks for nanoscale self-assembled systems, J. Mater. Chem. 2011, 21, 12181-12187.

[15]Y. Negishi, T. Nakazaki, S. Malola, S. Takano, Y. Niihori, W. Kurashige, S. Yamazoe, T. Tsukuda, H. Häkkinen, A Critical Size for Emergence of Nonbulk Electronic and Geometric Structures in DodecanethiolateProtected Au Clusters, J. Am. Chem. Soc. 2015, 137, 1206-1212.

[16]A. Moscatelli. Gold nanoparticles: Metallic up to a point, Nature Nanotechnol. 2015, DOI:10.1038/nnano.2015.16.

[17](a) M. Ali, I -Nan. Lin, The effect of the Electronic Structure, Phase Transition and Localized Dynamics of Atoms in the formation of Tiny Particles of Gold, http://arXiv.org/abs/1604.07144 (2016).

(b) M. Ali, I -Nan. Lin, The effect of the Electronic Structure, Phase Transition and Localized Dynamics of Atoms in the formation of Tiny Particles of Gold, J. Nanopart. Res. 2017, 19, 15.

[18]M. Ali, I -Nan. Lin, Geometric structure of gold tiny particles at varying precursor concentration and packing of their electronic structures into extended http://arXiv.org/abs/1604.07508 (2016).

[19] M. Ali, I -N. Lin, Controlling morphologystructure of particles via plastically driven geometric tiny particles and effect of photons on the structures under varying process conditions, http://arxiv.org/abs/1605.04408 (2016).

[20]M. Ali, I -N. Lin, Formation of tiny particles and their extended shapes-origin of physics and chemistry of materials, http://arxiv.org/abs/1605.09123 (2016).

[21] M. Ali, I -N. Lin, Dynamics of colloidal particles formation in processing different precursorselastically and plastically driven electronic states of atoms in lattice, http://arxiv.org/abs/1605.02296 (2016).

[22] M. Ali, I -Nan. Lin, Phase transitions and critical phenomena of tiny grains thin films synthesized in microwave plasma chemical vapor deposition and origin of v1 peak, http://arXiv.org/abs/1604.07152 (2016).

[23]M. Ali, M. Ürgen, Switching dynamics of morphology-structure in chemically deposited carbon films-a new insight, http://arxiv.org/abs/1605.00943 (2016).

[24] M. Ali, Atomic binding, geometric monolayer tiny particle, atomic deformation and one- 
dimensional http://arxiv.org/abs/1609.08047 (2016).

[25] M. Ali, Atoms deform or stretch, do not ionize, those having electronic transitions are the source of photonic current while inherently making terminals of inert gas atoms switch photonic current into photons, http://arxiv.org/abs/1611.05392 (2016).

[26] A. M. Marconnet, M. A. Panzer, K. E. Goodson, Thermal conduction phenomena in carbon nanotubes and related nanostructured materials, Rev. Mod. Phys. 2013, 85, 12951326.

[27] S. Nie, S. R. Emory, Probing Single Molecules and Single Nanoparticles by Surface-Enhanced Raman Scattering, Science 1997, 275, 11021106.

[28] J. Zhao, L. Yang, Structure Evolutions and Metallic Transitions in $\ln _{2} \mathrm{Se}_{3}$ Under High Pressure, J. Phys. Chem. C 2014, 118, 54455452.

[29] V. N. Manoharan, Colloidal matter: Packing, geometry, and entropy, Science 2015, 349, 1253751.

[30] J. Park, et al., 3D structure of individual nanocrystals in solution by electron microscopy, Science 2015, 349, 290-295.

[31]D. Jacobsson, et al., Interface dynamics and crystal phase switching in GaAs nanowires. Nature 2016, 531, 317-322.

[32]M. Ali, Structure evolution in atoms having phenomenon of electronic transitions, http://arxiv.org/abs/1611.01255(2016).

[33] T. Tuma, A. Pantazi, M. L. Gallo, E. Eleftheriou, Stochastic phase-change neurons. Nature Nanotech. 2016, 11, 693-699.

[34] J. Rensberg, et al., Active Optical Metasurfaces Based on Defect-Engineered Phase-Transition Materials, Nano Lett. 2016, 16, 1050-1055.

[35] Y. Suzuki, G. Cardone, D. Restrepo, P. D. Zavatteri, T. S. Baker, F. A. Tezcan, Self-assembly of coherently dynamic, auxetic, two-dimensional protein crystals, Nature 2016, 533, 369-373.

[36] C. H. J. Evers, J. A. Luiken, P. G. Bolhuis, W. K. Kegel, Self-assembly of microcapsules via colloidal bond hybridization and anisotropy, Nature 2016, 534, 364-368.
[37] I. R. Epstein, B. Xu, Reaction-diffusion processes at the nano- and microscales. Nature Nanotech. 2016, 11, 312-319.

[38]A. Azizi, Spontaneous Formation of Atomically Thin Stripes in Transition Metal Dichalcogenide Monolayers, Nano Lett. 2016, 16, 6982-6987.

[39] M. Mecklenburg, W. A. Hubbard, E. R. White, R. Dhall, S. B. Cronin, S. Aloni, B. C. Regan, Nanoscale temperature mapping in operating microelectronic devices, Science 2015, 347, 629-632.

[40]L. Ye, D. Hou, X. Zheng, Y. Yan, M. D. Ventra, Local temperatures of strongly-correlated quantum dots out of equilibrium, Phys. Rev. B 2015, 91, 205106-8.

[41]F. Menges, et al., Temperature mapping of operating nanoscale devices by scanning probe thermometry, Nat. Commun. 2016, 7:10874 doi: 10.1038/ncomms10874.

[42](a) D. Barredo, S. de Léséleuc, V. Lienhard, T. Lahaye, A. Browaeys, An atom-by-atom assembler of defect-free arbitrary $2 d$ atomic arrays, https://arxiv.org/abs/1607.03042, (2016) (b) D. Barredo, S. de Léséleuc, V. Lienhard, T. Lahaye, A. Browaeys, An atom-by-atom assembler of defect-free arbitrary twodimensional atomic arrays, Science 2016, 354, 1021-1023.

[43](a) M. Endres, et al., Cold Matter Assembled Atom-by-Atom, https://arxiv.org/abs/1607.03044, (2016). (b) M. Endres, et al., Atom-by-atom assembly of defect-free one-dimensional cold atom arrays, Science 2016, 354, 1024-1027.

[44] S. Kawai, et al., Van der Waals interactions and the limits of isolated atom models at interfaces, Nat. Commun. (2016) DOI: 10.1038/ncomms11559.

[45] A. Ambrosetti, N. Ferri, R. A. DiStasio Jr., A. Tkatchenko, Wavelike charge density fluctuations and van der Waals interactions at the nanoscale, Science 2016, 351, 1171-1176.

[46]A. Boltasseve, V. M. Shalaev, All that glitters need not be gold, Science 2015, 347, 13081310. 


\section{Author's biography:}

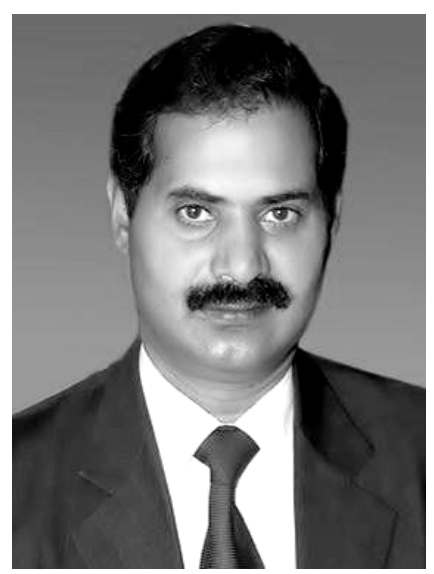

Mubarak Ali graduated from University of the Punjab with B.Sc. (Phys\& Maths) in 1996 and M.Sc. Materials Science with distinction at Bahauddin Zakariya University, Multan, Pakistan (1998); thesis work completed at Quaid-i-Azam University Islamabad. He gained Ph.D. in Mechanical Engineering from Universiti Teknologi Malaysia under the award of Malaysian Technical Cooperation Programme (MTCP;2004-07) and postdoc in advanced surface technologies at Istanbul Technical University under the foreign fellowship of The Scientific and Technological Research Council of Turkey (TÜBITAK; 2010). He completed another postdoc in the field of nanotechnology at Tamkang University Taipei (2013-2014) sponsored by National Science Council now M/o Science and Technology, Taiwan (R.O.C.). Presently, he is working as Assistant Professor on tenure track at COMSATS Institute of Information Technology, Islamabad campus, Pakistan (since May 2008) and prior to that worked as assistant director/deputy director at M/o Science \& Technology (Pakistan Council of Renewable Energy Technologies, Islamabad; 2000-2008). He was invited by Institute for Materials Research (IMR), Tohoku University, Japan to deliver scientific talk on growth of synthetic diamond without seeding treatment and synthesis of tantalum carbide. He gave several scientific talks in various countries. His core area of research includes materials science, condensed-matter physics \& nanotechnology. He was also offered the merit scholarship (for PhD study) by the Government of Pakistan but he couldn't avail. He is author of several articles published in various periodicals (https://scholar.google.com.pk/citations?hl=en\&user=UYjuhDwAAAAJ) and also a book.

(C) 2017 by the authors; licensee Preprints, Basel, Switzerland. This article is an open access article distributed under the terms and conditions of the Creative Commons by Attribution (CC-BY) license (http://creativecommons.org/licenses/by/4.0/). 